\title{
Provocative testing of the pulmonary circulation: advances and unresolved issues
}

\author{
Edmund M. Lau ${ }^{1}$ and Robert Naeije ${ }^{2}$
}

Affiliations: ${ }^{1}$ University of Sydney, Sydney, Australia. ${ }^{2}$ University of Brussels, Brussels, Belgium.

Correspondence: Robert Naeije, Dept of Cardiology, Erasme Campus CP 604, 808 Lennik road, B-1070 Brussels, Belgium. E-mail: rnaeijeaulb.ac.be

In this issue of the European Respiratory Journal, Oliveira et al. [1] from Harvard Medical School (Boston, MA, USA) report on rapid recovery of pulmonary vascular pressures after maximum incremental cycling exercise. The authors combined right heart catheterisation with concurrent cardiopulmonary exercise test in 95 patients referred for unexplained dyspnoea. 36 patients had precapillary pulmonary hypertension $(\mathrm{PH})$ during exercise, defined by the authors as displaying a peak exercise mean pulmonary artery pressure $(\mathrm{mPAP}) \geqslant 30 \mathrm{mmHg}$, a peak wedged pulmonary artery pressure (PAWP) $<20 \mathrm{mmHg}$ and a peak pulmonary vascular resistance (PVR) $\geqslant 1.5$ Wood units (WU). 28 patients had postcapillary PH during exercise defined by a peak PAWP $\geqslant 20 \mathrm{mmHg}$. 31 matched controls with normal resting mPAP were selected based on a maximum oxygen uptake $\geqslant 80 \%$ predicted in the absence of exercise $\mathrm{PH}$ based on the aforementioned criteria. Workload and oxygen uptake at the ventilatory threshold and at peak exercise were lower in the exercise PH patients, which confirm the relevance of exercise pulmonary haemodynamic measurements in unexplained dyspnoea. However, most of the exercise-induced increase in PAWP and $\mathrm{mPAP}$ was reversed within 2 min of recovery. The important message is that pulmonary haemodynamics have to be measured during, but not after, an exercise stress test for the diagnosis of exercise PH.

Provocative testing is used in many areas of medicine to uncover early-stage pathology. However, regarding early or latent $\mathrm{PH}$, experts remain utterly cautious. Reports and guidelines derived from the last World Symposium held in Nice (France) in 2013 state that exercise stress testing or volume loading of the pulmonary circulation is not encouraged because of insufficient evidence about the limits of normal and prognostic or therapeutic implications [2,3]. Yet the practice has been around since the early times of cardiac catheterisation [4] and significant progress has been made in recent years [5].

It is now well established that the upper limit of normal of mPAP during exercise is $30 \mathrm{mmHg}$ at a cardiac output $(\mathrm{CO})<10 \mathrm{~L} \cdot \mathrm{min}^{-1}$, which corresponds to a total pulmonary vascular resistance (TPR) (or mPAP/ $\mathrm{CO}$ ) of $3 \mathrm{WU}$ [5-9]. Meaningful noninvasive measurements are being reported by dedicated groups [5-7]. Furthermore, evidence is being gathered that higher than normal pulmonary artery pressure during exercise may be the cause of otherwise unexplained dyspnoea and may facilitate the early diagnosis of $\mathrm{PH}$ secondary to respiratory or left heart conditions $[6,7]$.

The cause of exercise PH is either an upstream transmission of increased PAWP, such as in heart failure with preserved or reduced ejection and left heart valvulopathies, or an increase in PVR, such as in pulmonary vascular disease, hypoxia or disturbed lung mechanics [4-8]. This differential diagnosis is often clinically straightforward, but precise measurement and interpretation of PAWP or left ventricular end-diastolic pressure measurements is paramount. The upper limit of normal of PAWP during exercise is generally thought to be between 15 and $20 \mathrm{mmHg}$ but higher values can be recorded in athletes and in

Received: April 302016 | Accepted: May 032016

Conflict of interest: None declared.

Copyright OERS 2016 
elderly subjects [10]. Oliveira et al. [1] considered $20 \mathrm{mmHg}$ as a reasonable upper limit of normal. However, a higher cut-off value of $25 \mathrm{mmHg}$ has been proposed for the diagnosis of heart failure [11, 12]. Likewise, for mPAP, a flow-corrected measure may be more appropriate. As TPR decreases during exercise by up to $25 \%$ [10], it is easy to predict normal PAWP/CO slopes of $<2 \mathrm{mmHg} \cdot \mathrm{L}^{-1} \cdot \mathrm{min}^{-1}$. This has indeed been measured but in limited size healthy control groups [11-13].

It is interesting that OlIVErRa et al. [1] consider PVR instead of TPR combined to increased pulmonary artery pressure in the diagnosis of exercise PH and actually retain a cut-off value of $1.5 \mathrm{WU}$ [1]. This PVR cut-off seems low, since a significant proportion of patients based on these criteria may not exceed a mPAP $>30 \mathrm{mmHg}$ together with TPR $>3 \mathrm{WU}$ during exercise. Definitions of $\mathrm{PH}$ have traditionally incorporated a "safety margin" to decrease the prevalence of false positives due to healthy outliers and lack of precision of measurements. This is why $\mathrm{PH}$ is defined by a $\mathrm{mPAP}>25 \mathrm{mmHg}$ at rest, while the upper limit of normal is $20 \mathrm{mmHg}$. One still does not know if a $\mathrm{mPAP}>30 \mathrm{mmHg}$ at a $\mathrm{CO}<10 \mathrm{~L} \cdot \mathrm{min}^{-1}$, corresponding to a TPR of $3 \mathrm{WU}$ is enough to minimise false positives across different ethnic populations and age groups. Conversely, the incorporation of a safety margin may also increase the likelihood of false-negative cases. In other words, any diagnostic thresholds assume a trade-off between sensitivity and specificity. For example, there was a recent noninvasive study showing higher exercise TPR values between 3 and 3.5 WU encountered in healthy sub-Saharan black African males [14]. An exercise TPR slightly above $3 \mathrm{WU}$ is also sometimes measured in invasively explored European healthy controls [8].

Measurements of pulmonary artery pressure and PAWP during exercise are technically challenging and are complicated by intrathoracic pressure swings [15]. It may then be advised to average the reading of pulmonary vascular pressure curves over several respiratory cycles instead of only at end-expiration [2]. This was actually done by Oliveira et al. [1] and the most recently updated European Respiratory Society/ European Society of Cardiology guidelines agree [3]. However, doubt remains about when to switch from one method to the other, and how relevant this is to symptoms and outcome.

Another increasingly popular stress test of the pulmonary circulation is fluid loading, which is mainly used to detect latent pulmonary venous hypertension in left heart diseases. Thus, any condition associated with altered left ventricular diastolic compliance or mitral valve stenosis will be associated with a rapid increase in PAWP when challenged with an increased systemic venous return [16]. Here also there has been debate about how to standardise a fluid challenge and what cut-off values for PAWP to consider. Fluid loading increases PAWP in healthy volunteers as a function of age, sex, amount infused and infusion rate [17]. While there is an emerging consensus to infuse $500 \mathrm{~mL}$ of saline in 5-10 min, some groups consider a PAWP of $15 \mathrm{mmHg}$ as a reasonable cut-off for a pathological response [18, 19]. However, a re-analysis of existing data gathered in healthy subjects and accumulating clinical experience [20] is drifting this cut-off value to $20 \mathrm{mmHg}$. It should not be overlooked that exercise increases systemic venous return as well. However, exercise may be more sensitive than volume loading to detect early pulmonary venous hypertension in patients with heart failure with preserved ejection fraction [21].

Where do we go from here? The report by Oliveira et al. [1] is an important step forward for the standardisation of provocative testing of the right ventricular-pulmonary circulation unit, but a lot remains to be done. While it is hopefully agreed that any test requires measurements while the system of interest is stressed, there are a series of other methodological issues that will require expert consensus standardisation. One of those may be body position, as some exercise tests are performed with the subject sitting on a bicycle, others in a tilted armchair convenient for echocardiography and others simply in the recumbent position of a catheterisation laboratory table. Body position certainly affects resting pulmonary haemodynamics since upright posture is associated with pulmonary vascular decruitment and reduced systemic venous return. However, body position is probably less important for exercise measurements, but more data are certainly needed. The exercise modality also matters, as a resistive component may be associated with an increased systemic vascular resistance or an increased intrathoracic pressure on Valsalva manoeuver, all affecting directly or indirectly pulmonary vascular pressures. Some centres measure mPAP as a function of workload, which is linearly related to $\mathrm{CO}$ but cannot be considered as a surrogate, as the range of CO responses to a given workload varies considerably [22]. One of the most urgent questions is that of end-expiratory versus average reading of pulmonary vascular pressure curves. Last but not least, there is a need for more validation of noninvasive approaches, as cardiac catheterisation cannot be generally used for screening or detection of early disease in minimally symptomatic patients.

In the meantime, Oliveira et al. [1] are to be congratulated for moving forward with extremely difficult experiments combining cardiac catheterisation and exercise testing. It is fascinating that there are still so many physiological questions to address for a better understanding and early diagnosis of different forms $\mathrm{PH}$, and that colleagues are ready to roll up their sleeves to solve still-pending methodological issues. No medical advance is possible without undisputed methodology. 


\section{References}

1 Oliveira RKF, Waxman AB, Agarwal $\mathrm{M}$, et al. Pulmonary haemodynamics during recovery from maximum incremental cycling exercise. Eur Respir J 2016; 48: 158-167.

2 Hoeper MM, et al. Definitions and diagnosis of pulmonary hypertension. J Am Coll Cardiol 2013; 62: Suppl. 25, D45-D50.

3 Galiè N, Humbert M, Vachiery J-L, et al. 2015 ESC/ERS Guidelines for the diagnosis and treatment of pulmonary hypertension. Eur Respir J 2015; 46: 903-975.

4 Wood P. Pulmonary hypertension with special reference to the vasoconstrictive factor. Br Heart J 1958; 20 : 557-570.

5 Naeije R, Vonk Noordegraaf A, Kovacs G. Exercise-induced pulmonary hypertension: at last! Eur Respir J 2015; 46: $583-586$.

6 Naeije R, Vanderpool R, Dhakal BP, et al. Exercise-induced pulmonary hypertension: physiological basis and methodological concerns. Am J Respir Crit Care Med 2013; 187: 576-583.

7 Lewis GD, Bossone E, Naeije R, et al. Pulmonary vascular hemodynamic response to exercise in cardiopulmonary diseases. Circulation 2013; 128: 1470-1479.

8 Herve P, Lau EM, Sitbon O, et al. Criteria for diagnosis of exercise pulmonary hypertension. Eur Respir J 2015; 46: 728-737.

9 Kovacs G, Avian A, Olschewski H. Proposed new definition of exercise pulmonary hypertension decreases false-positive cases. Eur Respir J 2016; 47: 1270-1273.

10 Kovacs G, Olschewski A, Berghold A, et al. Pulmonary vascular resistances during exercise in normal subjects: a systematic review. Eur Respir J 2012; 39: 319-328.

11 Borlaug BA, Nishimura RA, Sorajja $\mathrm{P}$, et al. Exercise hemodynamics enhance diagnosis of early heart failure with preserved ejection fraction. Circ Heart Fail 2010; 3: 588-595.

12 Andersen MJ, et al. Exercise hemodynamics in patients with and without diastolic dysfunction and preserved ejection fraction after myocardial infarction. Circ Heart Fail 2012; 5: 444-451.

13 Kovacs G, Olschewski A, Berghold A, et al. Pulmonary vascular resistances during exercise in normal subjects: a systematic review. Eur Respir J 2012; 39: 319-328.

14 Simaga B, Vicenzi M, Faoro V, et al. Pulmonary vascular function and exercise capacity in black sub-Saharan Africans. J Appl Physiol 2015; 119: 502-507.

15 Kovacs G, Avian A, Pienn M, et al. Reading pulmonary vascular pressure tracings. How to handle the problems of zero leveling and respiratory swings. Am J Respir Crit Care Med 2014; 190: 252-257.

16 Andersen MJ, Hwang SJ, Kane GC, et al. Enhanced pulmonary vasodilator reserve and abnormal right ventricular: pulmonary artery coupling in heart failure with preserved ejection fraction. Circ Heart Fail 2015; 8: 542-550.

17 Borlaug BA. Invasive assessment of pulmonary hypertension. Time for a more fluid approach? Circ Heart Fail 2014; $7: 2-4$.

18 Fujimoto N, Borlaug BA, Lewis GD, et al. Hemodynamic responses to rapid saline loading: the impact of age, sex, and heart failure. Circulation 2013; 127: 55-62.

19 Fox BD, Shimony A, Langleben D, et al. High prevalence of occult left heart disease in scleroderma-pulmonary hypertension. Eur Respir J 2013; 42: 1083-1091.

20 Robbins IM, Hemnes AR, Pugh ME, et al. High prevalence of occult pulmonary venous hypertension revealed by fluid challenge in pulmonary hypertension. Circ Heart Fail 2014; 7: 116-122.

21 Andersen MJ, Olson TP, Melenovsky V, et al. Differential hemodynamic effects of exercise and volume expansion in people with and without heart failure. Circ Heart Fail 2015; 8: 41-48.

22 Argiento P, Vanderpool RR, Mule M, et al. Exercise stress echocardiography of the pulmonary circulation: limits of normal and sex differences. Chest 2012; 590: 4279-4288. 